\title{
Shape optimization of hyperelastic rod
}

\author{
B. Rousselet \\ Universite de Nice \\ Departement de Mathematiques, Parc Valrose B.P. 71, 06108 Nice \\ Cedex 2, France. \\ J. Piekarski \\ Fundamental Technological Research Institute \\ ul. Swietokrzyska 21, 00-950 Warsaw, Poland. \\ A. Myslinski \\ System Research Institute \\ ul. Newelska 6, 01-447 Warsaw, Poland.
}

\begin{abstract}
The paper is concerned with an optimal design problem for hyperelastic rod. The function describing the position of a point at the line of rod cross-sections centroids in its reference configuration is variable subject to optimization. The necessary optimality condition is formulated. The continuation method with gradient descent method were employed to solve this problem numerically. The numerical results are provided.
\end{abstract}

\section{Keywords}

shape optimization, optimality condition, numerical methods

\section{INTRODUCTION}

The paper deals with the formulation of a necessary optimality condition for an optimal design problem of a hyperelastic rod as well as with the numerical solution of this problem. The rod is subjected to large displacement involving flexion, shear, torsion and longitudinal extension ( see Antman and Kenney (1981)). The equilibrium state of the rod is described by a system of two nonlinear coupled ordinary differential equations of the first order. In this model the function describing the position of the point at the line of centroids of cross-sections of the rod as well as the orthonormal directors are used to characterize the motion and deformation of the rod in an objective and intrinsic framework ( see Antman and Kenney (1981)). Moreover the nonlinear geometry of the rod is taken into account exactly. The existence of solutions to the rod problem was studied by Antman and Kenney (1981), Ciarlet (1986), LeTallec, Mani and Rochinha (1992).

The optimal design problem for hyperelastic rod consists in finding such reference configuration of the rod to minimize its compliance (see Haug, Choi and Komkov(1986), 
Rousselet(1992)) measured as a distance between current and reference configurations occupied by the rod loaded by a given body force. The function describing the position of a point at the line of centroids of cross-sections of the rod in its reference configuration is variable subject to optimization. It is assumed that this function is bounded and has bounded derivative.

The aim of this paper is to formulate the shape optimization problem for the highly nonlinear rod model and to derive a necessary optimality condition. We shall show Lipschitz continuity of the solution to the rod model with respect to the optimized variable. The design sensitivity analysis of the solution to the state system is performed and the directional derivative of the cost functional is calculated. Using this derivative a necessary optimality condition is formulated. The calculated derivative is employed as an element of descent direction finding procedure in numerical algorithm for solving this optimization problem. Numerical example is provided.

\section{KINEMATIC DESCRIPTION}

Consider a rod in a reference configuration. This configuration is specified by enough regular mapping $\varphi_{0}(\xi)$ and an orthonormal pair of vector functions $E_{1}(\xi)$ and $E_{2}(\xi)$ of the real variable $\xi \in[0, L]$. The mapping :

$\varphi_{0}: R \ni \xi \rightarrow \varphi_{0}(\xi) \in R^{3}$

defines the line of centroids of cross-sections of a slender three dimensional body called a rod Antman and Kelly (1981). The line of centroids is assumed to be an arbitrary curve. The cross-section $A(\xi)$ of the rod is defined as a plane passing through a point $\varphi_{0}(\xi) \in R^{3}$, $\xi \in[0, L]$ and normal to the vector $E_{3}(\xi)=E_{1}(\xi) \times E_{2}(\xi) . \varphi_{0}(\xi)$ is interpreted as the position in a reference configuration of the material point at the centroid line of the section $\varphi$ (see Antman and Kelly (1981), LeTallec, Mani and Rochinha (1992)). $E_{1}(\xi)$ and $E_{2}(\xi)$ characterize the reference configuration of the section $\xi$. In the reference configuration the rod occupies the domain : $\Omega_{0}=\left\{X \in R^{3}: X=\varphi_{0}(\xi)+\sum_{i=1}^{2} \tilde{X}_{i} E_{i}(\xi), 0 \leq \xi \leq\right.$ $\left.L,\left(\tilde{X}_{1}, \tilde{X}_{2}\right) \in A(\xi)\right\}$ Note that $\left\{E_{i}(\xi)\right\}, i=1,2,3$ is a moving orthonormal frame in the reference configuration.

The rod can suffer flexure, torsion, shear and longitudinal extension (see Antman and Kelly (1981), LeTallec, Mani and Rochinha(1992)). After deformation the current configuration of the rod is characterized by a position vector function $\varphi(\xi)$ of the line of centroids and an orthonormal pair of vectors $t_{1}(\xi)$ and $t_{2}(\xi)$ defining the cross-section of the deformed rod. The functions $\varphi, t_{1}, t_{2}$ depending on $\xi \in[0, L]$ have similar interpretation as $\varphi_{0}, E_{1}, E_{2}$ (see Antman and Kelly (1981)). The cross-section $A(\xi)$ of the deformed rod is defined as a plane passing through a point $\varphi(\xi) \in R^{3}, \xi \in[0, L]$ and normal to the vector $t_{3}(\xi)=t_{1}(\xi) \times t_{2}(\xi)$. In the current configuration each material point of the deformed rod occupies the position $\mathrm{x}(\mathrm{X})$. The set of points $x$ is called the set of admissible configurations and is determined by : $\Omega=\left\{x \in R^{3}: x=\varphi(\xi)+\sum_{i=1}^{2} X_{i} t_{i}(\xi), 0 \leq \xi \leq\right.$ $\left.L,\left(X_{1}, X_{2}\right) \in A(\xi)\right\}$ where $\varphi:[0, L] \ni \xi \rightarrow \varphi(\xi) \in R^{3}$ defines the position of the line of centroids after deformation and $\left\{t_{i}(\xi)\right\}, i=1,2,3$ is an orthonormal moving frame in the current configuration.

We shall assume that the deformed images of the two cross-sections are incapable of touching within the rod (see Ciarlet(1988)), i.e., $\operatorname{det}\left(t_{1}(\xi), t_{2}(\xi), t_{3}(\xi)\right)>0 \quad \forall \xi \in[0, L]$. 
Moreover the deformation $x(X)$ is assumed to satisfy the kinematic boundary conditions imposed to the rod. Assume these conditions are :

$\varphi(0)=\varphi_{0} \quad \varphi(L)=\varphi_{0}(L)=\varphi_{L}, \quad t_{i}(0)=E_{i}(0)$ for $i$ given in $\{1,2,3\}$

where $\varphi_{0}, \varphi_{L}$ and $E_{i}(0), i=1,2,3$ are given vectors. The condition (2) imply the rod is fixed at both ends as well as the rod can rotate around $E_{i}(0)$.

\section{VARIATIONAL FORMULATION}

Let $E=R^{3}$ and $L^{p}(0, L), 1 \leq p<\infty$, denotes the space of integrable functions (see Ciarlet(1988)). We denote by $W^{m, p}(0, L), m \geq 1,1 \leq p<\infty$ the Sobolev space (see Ciarlet(1988)). By K we denote the set of kinematically admissible deformation fields :

$$
\begin{array}{r}
K=\left\{\left\{\varphi, t_{1}, t_{2}, t_{3}\right\}=\left\{\varphi, t_{i}\right\} \in W^{1, p}\left(0, L ; E^{4}\right): \varphi(0)=\varphi_{0}, \varphi(L)=\varphi_{L}, t_{i}(0)=E_{i}(0)\right. \\
\text { for } i \in\{1,2,3\} ; t_{i}(\xi) t_{j}(\xi)=\delta_{i j} \forall i, j=1,2,3, \forall \xi \in[0, L] \\
\left.\qquad \operatorname{det}\left(t_{1}(\xi), t_{2}(\xi), t_{3}(\xi)\right)>0 \forall \xi \in[0, L]\right\}
\end{array}
$$

By $E(.,):. K \rightarrow R$ we denote potential energy functional given by Antman and Kelly(1981), Ciarlet(1988), LeTallec, Mani and Rochinha(1992) :

$E\left(\varphi, t_{i}\right)=\int_{0}^{L} w\left(\xi, \chi^{j}\left(t_{i}\right), \Gamma^{k}\left(\varphi, t_{i}\right)\right) d \xi-\int_{0}^{L}\left(\tilde{n} \varphi+f t_{i}\right) d \xi$

where $\bar{n} \in L^{p^{*}}(0, L ; E)$ and $f \in L^{p^{*}}\left(0, L ; E^{3}\right)$ are given, $p^{*}=\frac{p}{p-1}$ and $w$ denotes the stored energy function (see Rousselet, Piekarski, Myslinski(1995)). $\chi$ and $\Gamma$ denote the strain measures (see Antman and Kelly(1981)), LeTallec, Mani and Rochinha(1992)). The equilibrium state of the rod is characterized by :

Find $\left\{\varphi, t_{i}\right\} \in K$ such that : $E\left(\varphi, t_{i}\right) \leq E\left(z, d_{i}\right) \forall\left\{z, d_{i}\right\} \in K$

Let us denote by $d K\left(\varphi, t_{i}\right)$ the space of kinematically admissible variations (see LeTallec, Mani and Rochinha(1992)).

$$
\begin{array}{r}
d K\left(\varphi, t_{i}\right)=\left\{\left\{\delta \varphi, \delta t_{i}\right\} \in W^{1, p}\left(0, L ; E^{4}\right): \delta \varphi(0)=\delta \varphi(L)=0, \delta t_{i}(0)=0\right. \\
\text { for } \left.i \in\{1,2,3\} ; \quad \exists \theta \in W^{1, p}(0, L ; E), \delta t_{i}=\theta \times t_{i} \forall i=1,2,3\right\}
\end{array}
$$

$d K\left(\varphi, t_{i}\right)$ is the space of all tangent vectors to the set $\mathrm{K}$ at a point $\left\{\varphi, t_{i}\right\} . \delta($.$) may be$ interpreted as a differentiation operator on $\mathrm{K}$ into a tangent direction to $\mathrm{K}$ (see Antman and Kelly(1981), LeTallec, Mani and Rochinha(1992)). Let us introduce the following forms :

$$
\left[W^{\mathbf{1}, p}\left(0, L ; E^{\mathbf{4}}\right)\right]^{2} \ni \tilde{b}\left(\varphi, t_{i}, \delta \varphi, \delta t_{i}\right)=\int_{0}^{L}\left\{n\left[(\delta \varphi)^{\prime}-\theta \times(\varphi)^{\prime}\right]+m \theta^{\prime}\right\} d s \rightarrow R
$$


$W^{1, p}\left(0, L ; E^{4}\right) \ni l\left(\delta \varphi, \delta t_{i}\right)=\int_{0}^{L}\left(\bar{n} \delta \varphi+f_{i} \delta t_{i}\right) d s \rightarrow R$

wher $n$ and $m$ denote resultant contact force and moment respectively. Assuming the solutions to (5) are smooth enough the problem (5) is formally equivalent to the following system (see Ciarlet(1988)) : Find $\left\{\varphi, t_{i}\right\} \in K$ satisfying

$\tilde{b}\left(\varphi, t_{i}, \delta \varphi, \delta t_{i}\right)=l\left(\delta \varphi, \delta t_{i}\right) \quad \forall\left\{\delta \varphi, \delta t_{i}\right\} \in d K\left(\varphi, t_{i}\right)$

Problem (9) is formulated in the current configuration. From numerical point of view (see LeTallec, Mani and Rochinha(1992)) it is convenient to consider this problem transformed to the reference configuration. Using the mapping transforming $\left\{E_{i}\right\}$ onto $\left\{t_{i}\right\}$ (see Rousselet, Piekarski and Myslinski(1995)) we can write problem (9) in the reference configuration.

Lemma 31 Problem (g) is equivalent to the following variational problem: Find $\left\{\varphi, t_{i}\right\}$ $\in K$ satisfying

$b\left(\varphi, t_{i}, \delta \varphi, \delta t_{i}\right)=l\left(\delta \varphi, \delta t_{i}\right) \quad \forall\left\{\delta \varphi, \delta t_{i}\right\} \in d K\left(\varphi, t_{i}\right)$

where the form $b(., ., .,):.\left[W^{1, p}\left(0, L ; E^{4}\right)\right]^{2} \rightarrow R$ is given by $b\left(\varphi, t_{i}, \delta \varphi, \delta t_{i}\right)=\int_{0}^{L}(N \delta \Gamma+$ $M \delta \chi) d s$ where $N$ and $M$ denote the contact force and moment in the reference configuration and $\delta \Gamma$ and $\delta \chi$ denote the increments of strains in the reference configuration.

Proof. Proof is given in Rousselet, Piekarski and Myslinski(1995).

The existence of global and local solutions to the state system (10) was studied by Ciarlet(1988).

\section{FORMULATION OF THE OPTIMIZATION PROBLEM}

Let $\varphi_{0}=\varphi_{0}(\xi)$ defined by (1) be the variable subject to optimization. $\varphi_{0}$ is assumed to satisfy :

$c_{1} \leq \varphi_{0}(\xi) \leq c_{2}, \quad\left|\frac{d \varphi_{0}}{d \xi}\right| \leq c_{3},\left|\frac{d^{2} \varphi_{0}}{d \xi^{2}}\right| \leq c_{4}, \quad \int_{A(\xi)} \varphi_{0}(\xi) d \xi=c_{5}$

where $c_{1}, c_{2}, c_{3}, c_{4}, c_{5}$ are given positive constants. By $U_{a d}$ we denote the set of admissible designs :

$U_{a d}=\left\{\varphi_{0} \in C^{2,1}(0, L ; E): \varphi_{0}\right.$ satisfies $\left.(11)\right\}$

$C^{2,1}$ denotes a space of Lipschitz continuous functions having Lipschitz continuous first and second order derivatives (see Ciarlet(1988)). $U_{a d}$ is assumed to be nonempty. In order to underline the dependence of the solution $\left\{\varphi, t_{i}\right\} \in K \cap W^{2, p}(0, L)$ to the system $(10)$ on $\varphi_{0} \in U_{a d}$ we shall write $: b\left(\varphi_{0}, \varphi, t_{i}, \delta \varphi, \delta t_{i}\right)$ for $b\left(\varphi, t_{i}, \delta \varphi, \delta t_{i}\right), l\left(\varphi_{0}, \delta \varphi, \delta t_{i}\right)$ for $l\left(\delta \varphi, \delta t_{i}\right)$. 
From (see Rousselet, Piekarski, and Myslinski(1995)) it follows that for a given point $\varphi_{0} \in U_{a d}$, problem (10) has a unique solution $\left\{\varphi, t_{i}\right\} \in K \cap W^{2, p}(0, L), p \geq 2$ in a neighborhood of the linearization point $\left\{\varphi_{0}, E_{i}\right\} \in K$. We shall consider the following optimization problem: Find $\varphi_{0} \in U_{\text {ad }}$ minimizing the cost functional :

$J\left(\varphi_{0} ; \varphi, t_{i}\right)=\int_{0}^{L}\left\{\left(\varphi-\varphi_{0}\right)^{2}+\left(t_{i}-E_{i}\right)^{2}\right\} d s$

on the set $U_{a d} .\left\{\varphi, t_{i}\right\} \in K \cap W^{2, p}(0, L)$ is a solution to the system (10).

The cost functional (13) approximates the compliance of the rod (see Haug, Choi and Komkov(1986)). If $\varphi, t_{i}, i=1,2,3$ are Lipschitz continuous functions with respect to $\varphi_{0} \in$ $U_{a d}$ then from Weierstrass Theorem follows the existence of optimal solution $\hat{\varphi}_{0} \in U_{a d}$ to the problem (13).

We shall calculate the directional derivative of the cost functional (13). The directional derivative $d J\left(\varphi_{0}, \delta \varphi_{0}\right)$ of the cost functional (13) at a point $\varphi_{0} \in U_{a d}$ in the direction $\delta \varphi_{0} \in U_{a d}$ is given by :

$$
\begin{array}{r}
d J\left(\varphi_{0}, \delta \varphi_{0}\right)=2 \int_{0}^{L}\left\{\left(\varphi-\varphi_{0}\right)\left(\frac{\partial \varphi}{\partial \varphi_{0}}-1\right) \delta \varphi_{0}+\left(t_{i}-E_{i}\right)\left(\frac{\partial t_{i}}{\partial \varphi_{0}}-\frac{\partial E_{i}}{\partial \varphi_{0}}\right) \delta \varphi_{0}\right\} d s+ \\
\int_{0}^{L}\left[\left(\varphi-\varphi_{0}\right)^{2}+\left(t_{i}-E_{i}\right)^{2}\right] E_{3} \frac{d \delta \varphi_{0}}{d s} d s
\end{array}
$$

\section{DESIGN SENSITIVITY ANALYSIS OF SOLUTIONS TO THE STATE SYSTEM}

Recall from Rousselet, Piekarski and Myslinski(1995) that $\left\{\varphi, t_{i}\right\} \in K \cap W^{2, p}(0, L)$ is a unique local solution to the nonlinear system (10) in a neighborhood of a linearization point $\left\{\varphi_{0}, E_{i}\right\} \in K$.

Assuming that the solution $\left\{\varphi, t_{i}\right\} \in K \cap W^{2, p}(0, L)$ is sufficiently close to the linearization point $\left\{\varphi_{0}, E_{i}\right\} \in K$, from Continuity Theorem (see Ciarlet(1988)) it follows that the coercivity condition for the linearized form $a$ of the form $b$ still holds at a point $\left\{\varphi, t_{i}\right\} \in K \cap W^{2, p}(0, L)$, i.e., we have : there exists constant $\alpha>0$ such that for all $\left\{\delta \varphi, \delta t_{i}\right\} \in d K\left(\varphi_{0}, E_{i}\right)$ the following condition holds :

$a\left(\varphi_{0}, \varphi, t_{i}, \delta \varphi, \delta t_{i}, \delta \varphi, \delta t_{i}\right) \geq \alpha\left(\|\delta \varphi\|_{W^{1,2}(0, L)}^{2}+\left\|\delta t_{i}\right\|_{W^{1,2}(0, L)}^{2}\right)$

where $a$ denoting the linearized form of the form $b$ is given by Rousselet, Piekarski and Myslinski(1995). Using (15) we are able to prove :

LEMma 51 If $\left\{\varphi, t_{i}\right\} \in K \cap W^{2, p}(0, L)$ is a unique local solution to the system (10) then there exists the Frechet dertivative of the mapping

$U_{a d} \ni \varphi_{0} \rightarrow\left(\varphi, t_{i}\right) \in K$

at a point $\left\{\varphi, t_{i}\right\} \in K \cap W^{2, p}(0, L)$ in a direction $\delta \varphi_{0} \in U_{a d}$. 
Proof. From Rousselet, Piekarski and Myslinski(1995) it follows that a point $\left\{\varphi, t_{i}\right\} \in$ $K \cap W^{2, p}(0, L)$ is a unique solution to the system (10). Let us calculate the derivatives of the form (10) with respect to $\varphi$ and $t_{i}$, respectively, at a point $\left\{\varphi, t_{i}\right\} \in K \cap W^{2, p}(0, L)$ in a direction $\left\{\tilde{\delta} \varphi, \tilde{\delta} t_{i}\right\} \in d K\left(\varphi, t_{i}\right)$. These derivatives are given by :

$$
\begin{gathered}
\frac{\partial b}{\partial \varphi}\left(\varphi_{0}, \varphi, t_{i}, \delta \varphi, \delta t_{i}\right) \tilde{\delta} \varphi=\int_{0}^{L}\left\{c_{i}\left(\frac{d \tilde{\delta} \varphi}{d s} t_{i}\right)\left[t_{i}\left(\frac{d \delta \varphi}{d s}-\theta \times \frac{d \varphi}{d s}\right)\right]\left[c_{i}\left(\frac{d \varphi}{d s} t_{i}\right)-\bar{c}_{i}\right] \frac{d \tilde{\delta} \varphi}{d s} \delta t_{i}\right\} d s \\
\frac{\partial b}{\partial t_{i}}\left(\varphi_{0}, \varphi, t_{i}, \delta \varphi, \delta t_{i}\right) \tilde{\delta} t_{i}=\int_{0}^{L}\left\{c_{i}\left(\frac{d \varphi}{d s} \tilde{\delta} t_{i}\right)\left[t_{i}\left(\frac{d \delta \varphi}{d s}-\theta \times \frac{d \varphi}{d s}\right)\right]+d_{i}\left(\frac{d \tilde{\theta}}{d s} t_{i}\right)\left(\frac{d \theta}{d s} t_{i}\right)\right\}+ \\
\int_{0}^{L}\left\{\left[c_{i}\left(\frac{d \varphi}{d s} t_{i}\right)-\bar{c}_{i}\right]\left[\frac{d \delta \varphi}{d s} \tilde{\delta} t_{i}-\left(\theta \times \frac{d \varphi}{d s}\right) \tilde{\delta} t_{i}\right]+\frac{1}{2} e_{i k l} d_{i}\left(\frac{d t_{k}}{d s} t_{l}-\frac{d E_{k}}{d s} E_{l}\right)\left(\frac{d \theta}{d s} \tilde{\delta} t_{i}\right)\right\} d s
\end{gathered}
$$

The derivatives (17) and (18) are linear and bounded. Moreover from (15) it follows positiv definiteness of the derivatives (17) and (18) in the space $W^{1,2}(0, L)$. Hence by implicit function theorem (see Ciarlet(1988)) as well as the regularity of solutions to the system (10) follows the Frechet differentiability of the mapping (16).

Assume that the curve $\varphi_{0} \in U_{a d}$ is perturbed into a curve $\left(\varphi_{0}+\delta \varphi_{0}\right) \in U_{a d}$. We derive the design sensitivity of the state system (10). Using Lemma 5.1 and differentiating the system (10) with respect to $\varphi_{0} \in U_{a d}$ at a point $\left\{\varphi, t_{i}\right\} \in K$ in a direction $\delta \varphi_{0} \in U_{a d}$ we obtain :

$$
\begin{array}{r}
\frac{\partial b}{\partial \varphi_{0}}\left(\varphi_{0}, \varphi, t_{i}, \delta \varphi, \delta t_{i}\right) \delta \varphi_{0}+\frac{\partial b}{\partial \varphi}\left(\varphi_{0}, \varphi, t_{i}, \delta \varphi, \delta t_{i}\right) \frac{\partial \varphi}{\partial \varphi_{0}} \delta \varphi_{0}+ \\
\frac{\partial b}{\partial t_{i}}\left(\varphi_{0}, \varphi, t_{i}, \delta \varphi, \delta t_{i}\right) \frac{\partial t_{i}}{\partial \varphi_{0}} \delta \varphi_{0}=\frac{\partial l}{\partial \varphi_{0}}\left(\varphi_{0}, \delta \varphi, \delta t_{i}\right) \delta \varphi_{0} \quad \forall\left\{\delta \varphi, \delta t_{i}\right\} \in d K\left(\varphi, t_{i}\right)
\end{array}
$$

The derivatives $\frac{\partial b}{\partial \varphi}\left(\varphi_{0}, \varphi, t_{i}, \delta \varphi, \delta t_{i}\right)$ and $\frac{\partial b}{\partial t_{i}}\left(\varphi_{0}, \varphi, t_{i}, \delta \varphi, \delta t_{i}\right)$ are given by (17) and (18) respectively. The derivatives with respect to $\varphi_{0}$ are calculated in Rousselet, Piekarski and Myslinski(1995).

\section{NECESSARY OPTIMALITY CONDITION}

Using (19) and formulae in Rousselet, Piekarski and Myslinski(1995) we shall calculate the derivative (14) explicitly. Let us introduce an adjoint state $\left\{\delta \lambda, \delta \varepsilon_{i}\right\} \in d K\left(\varphi, t_{i}\right)$ defined as the solution to the following system :

$$
\begin{array}{r}
\frac{\partial b}{\partial \varphi}\left(\varphi_{0}, \varphi, t_{i}, \delta \lambda, \delta \varepsilon_{i}\right) \delta \varphi+\frac{\partial b}{\partial t_{i}}\left(\varphi_{0}, \varphi, t_{i}, \delta \lambda, \delta \varepsilon_{i}\right) \delta t_{i}= \\
-2 \int_{0}^{L}\left\{\left(\varphi-\varphi_{0}\right) \delta \varphi+\left(t_{i}-E_{i}\right) \delta t_{i}\right\} d s \quad \forall\left\{\delta \varphi, \delta t_{i}\right\} \in d K\left(\varphi, t_{i}\right)
\end{array}
$$


The derivatives $\frac{\partial b}{\partial \varphi}$ and $\frac{\partial b}{\partial t_{i}}$ in (20) are determined by (17) and (18) respectively. From (15) and Lax-Milgram Lemma (see Ciarlet(1988)) follows the existence of a unique solution $\left\{\delta \lambda, \delta \varepsilon_{i}\right\} \in d K\left(\varphi, t_{i}\right)$ to the adjoint system $(20)$.

Using (14), (17), (18), (20) as well as Rousselet, Piekarski and Myslinski(1995) we can calculate the directional derivative of the cost functional $(13)$ :

$d J\left(\varphi_{0}, \delta \varphi_{0}\right)=\frac{\partial b}{\partial \varphi_{0}}\left(\varphi_{0}, \varphi, t_{i}, \delta \lambda, \delta \varepsilon_{i}\right) \delta \varphi_{0}-\frac{\partial l}{\partial \varphi_{0}}\left(\varphi_{0}, \delta \lambda, \delta \varepsilon_{i}\right) \delta \varphi_{0}+$
$2 \int_{0}^{L}\left\{\left(\varphi-\varphi_{0}\right) \delta \varphi_{0}+\left(t_{i}-E_{i}\right) \frac{\partial E_{i}}{\partial \varphi_{0}} \delta \varphi_{0}\right\} d s+\int_{0}^{L}\left[\left(\varphi-\varphi_{0}\right)^{2}+\left(t_{i}-E_{i}\right)^{2}\right] E_{3} \frac{d \delta \varphi_{0}}{d s} d s$

where the derivatives with respect to $\varphi_{0}$ are determined in Rousselet, Piekarski and Myslinski(1995).

LEMMA 61 If $\hat{\varphi}_{0} \in U_{a d}$ is an optimal solution to the problem (13), then there exist Lagrange multipliers $\mu_{1} \in C^{1,1}(0, L), \mu_{2} \in C^{2,1}(0, L), \mu_{3} \in\left[C^{2,1}(0, L)\right]^{*}$ such that for all $\delta \varphi_{0} \in C^{2,1}(0, L ; E)$ satisfying $c_{1} \leq \hat{\varphi}_{0}+\delta \varphi_{0} \leq c_{2}$ where $c_{1}, c_{2}$ are the constants as in (11), the following condition holds :

$\frac{\partial J}{\partial \varphi_{0}}\left(\hat{\varphi}_{0}\right) \delta \varphi_{0}+\operatorname{sgn}\left(\frac{d \hat{\varphi}_{0}}{d \xi}\right) \int_{0}^{L} \mu_{1} \frac{d \delta \varphi_{0}}{d \xi} d \xi+\operatorname{sgn}\left(\frac{d^{2} \hat{\varphi}_{0}}{d \xi^{2}}\right) \int_{0}^{L} \mu_{2} \frac{d^{2} \delta \varphi_{0}}{d \xi^{2}} d \xi+\int_{A(\xi)} \mu_{3} \delta \varphi_{0} d \xi \geq 0(22)$

where the derivative $\frac{\partial J}{\partial \varphi_{0}}\left(\varphi_{0}\right) \delta \varphi_{0}$ is given by (21) and the function sgn(.) is defined by : $\operatorname{sgn}(x)=1$ if $x>0, \operatorname{sgn}(x)=0$ if $x=0, \operatorname{sgn}(x)=-1$ if $x<0$.

Proof. Proof is standard (see Ciarlet (1988)).

\section{NUMERICAL RESULTS}

The optimization problem (13) was solved using the descent method (see Haug, Choi and Komkov(1986)). The centroid line $\varphi_{0}$, the design parameter, was interpolated by cubic Hermit spline. The calculated directional derivative (21) was used in the descent algorithm. The state problem (10) is solved using the finite element method. The line of centroids $\varphi$ as well as directors $t_{i}$ were discretized using the linear finite elements (see Rousselet, Piekarski and Myslinski(1995), LeTallec, Mani and Rochinha(1992)). The one point Gauss quadrature formula was used.

The continuation method was used to solve the nonlinear system (10) where the predictor colinear with the variable increment in the previous iteration was used and the orthogonal plane method as the correction method was employed.

As an example we considered $2 \mathrm{D}$ arch with rectangular, constant cross-section. The centroid line of this arch is assumed to occupy in the initial undeformed configuration the line given by parabola

$y(\xi)=-(\xi-0.5)(\xi+0.5)$ where $\xi \in[a, b]$

The numerical data are : $a=-0.5, b=0.5$. The line (23) was interpolated using 5 
Table 1 Initial and optimal shapes of arch. 1 - initial non-loaded form, 2 - optimal non-loaded form for $f_{2}=2.5 e+04,3$ - optimal non-loaded form for $f_{1}=10$

\begin{tabular}{cccccccc}
\hline$\xi$ & -0.50 & -0.40 & -0.20 & 0.00 & 0.20 & 0.40 & 0.50 \\
\hline 1 & 0.00 & 0.08 & 0.20 & 0.25 & 0.20 & 0.08 & 0.00 \\
\hline 2 & 0.00 & 0.11 & 0.36 & 0.45 & 0.36 & 0.11 & 0.11 \\
\hline 3 & 0.00 & 0.39 & 0.61 & 0.75 & 0.61 & 0.39 & 0.11 \\
\hline
\end{tabular}

nodes with coordinates : $x=-0.5,-0.3,0.0,0.3,0.5$. The nodal points of the discretized arch are allowed to move vertically only (see Haug, Choi and Komkov(1986)). To solve the system $(10)$ the segment $[a, b]$ has been divided into 20 elements. The performance of the optimization algorithm is measured by : $w_{1}=J\left(\hat{\varphi}_{0}\right) / J\left(\varphi_{0}^{i n i}\right), \quad w_{2}=\left\|d J\left(\hat{\varphi}_{0}\right)\right\| / \|$ $d J\left(\varphi_{0}^{i n i}\right) \|$ where $\hat{\varphi}_{0}$ and $\varphi_{0}^{i n i}$ are optimal and initial configuration of the arch centroid line, respectively, $J$ is a cost functional defined by $(13), d J($.$) is determined by (21). In$ the formula for $w_{2}$ the euclidean norm is used.

The obtained result is presented in Table 1 . The computations were performed for the following values of the concentrated force $f: f_{1}(\xi)=10$ linear case, $f_{2}(\xi)=$ $2.5 \cdot 10^{4}$ nonlinear case. Table 1 presents the initial, non-loaded form of the arch in the reference configuration (23) as well as the optimal non-loaded forms of the arch with the $f_{1}$ and $f_{2}$ value of forces, respectively. Note that the maximal height of the optimal shape arch for the force $f_{2}$ is lower than the arch height for the load $f_{1}$. Moreover the shape near the end points for both loads has been significantly changed in comparison to the initial form. In the linear case : $w_{1}=0.139, w_{2}=4.1 \cdot 10^{-2}$. For the nonlinear arch these values are $: w_{1}=0.385, w_{2}=2.61 \cdot 10^{-4}$.

\section{REFERENCES}

Antman, S.S. and Kenney, C.S. (1981) Large Buckled States of Nonlineary Elastic Rods under Thorsion, Thrust and Gravity. Archive for Rational Mechanics and Analysis, 76, $289-338$.

Ciarlet, Ph. (1988) Mathematical Elasticity. Vol 1: Three Dimensional Elasticity. NorthHolland, Amsterdam.

Haug, E.J., Choi, K.K. and Komkov, V. (1986) Design Sensitivity Analysis of Structural Systems. Academic Press, New York.

LeTallec, P., Mani, S. and Rochinha, F.A. (1992) Finite Element Computation of Hyperbolic Rods in Large Displacement. Mathematical Modelling and Numerical Analysis, 26, $595-625$.

Rousselet, B. (1992) A Finite Strain Rod Model and its Design Sensitivity. Mechanical Structures and Machinery, 20, No 4.

Rousselet, B., Piekarski, J. and Myslinski, A. (1995) Design Sensitivity for Hyperelastic Rod in Large Displacements with respect to its Midcurve. Report. 